\title{
A Comparison of Pharmacotherapy in Inpatients with Major Depressive Disorders Between Patients With Single Episodes and Patients With Recurrent Episodes
}

\author{
Akeo Kurumaji, M.D., Ph.D., ${ }^{*}$ Kenji Narushima, M.D., Ph.D., ${ }^{*}$ Mitsuhiro Takeda, M.D., ${ }^{* *}$ Daisuke \\ Jitoku, M.D., Ph.D., ${ }^{*}$ Hotsumi Kyono, M.D., ${ }^{* *}$ Mizue Hobo, M.D., ${ }^{* * *}$ Hiroo Mitsusada, M.D., ${ }^{* *}$ \\ Masaki Nishida, M.D., Ph.D., ${ }^{*}$ Hidenori Atsuta, M.D., ${ }^{* *}$ Shin-ichiro Tamai, M.D., ${ }^{* *}$ Daisuke Takagi, \\ M.D., ${ }^{* *}$ Munehisa Fujita, M.D., ${ }^{* *}$ Koutarou Kawamata, M.D., ${ }^{* *}$ Shouko Okuzumi, M.D., ${ }^{* *}$ Kouhei \\ Hino, M.D., ${ }^{* *}$ Keita Tsutsui, M.D., ${ }^{* *}$ and Toru Nishikwa, M.D., Ph.D.* \\ *Section of Psychiatry and Behavioral Sciences, Tokyo Medical and Dental \\ University Graduate School, Tokyo 113-8519, Japan. \\ **Department of Psychiatry, Medical Hospital, Tokyo Medical \\ and Dental University, Tokyo 113-8519, Japan. \\ *** Department of Sleep Modulatory Medicine, Tokyo Medical and Dental \\ University Graduate School, Tokyo 113-8519, Japan.
}

\begin{abstract}
Purpose: Antidepressants possessing a wide range of pharmacological properties are available for the daily treatment of major depressive disorders (MDD). However, MDD patients with a partial response or a resistance to standard antidepressant therapy need to be treated with adjunctive psychotropic drugs such as mood stabilizers or atypical antipsychotics in order to achieve full remission. Investigation of combination pharmacotherapy in actual clinical practice could provide an insight into the features of adjunctive medications.

Methods: The subjects of the study were MDD inpatients who were admitted from April 2010 to March 2013. The demographic and clinical data and prescription profiles for the psychotropic drugs at discharge were obtained from the medical charts of the patients. A statistical analysis of the data was conducted to clarify the difference between a single episode and recurrent episodes of MDD.

Results: The adjunctive medication prescription rate for patients with recurrent episodes was significantly higher for antipsychotics, mood stabilizers and hypnotic drugs than that for patients with a single episode, even though no statistically significant differences were found between the two types of patients in their background characteristics. In addition, the proportion of patients who received prescriptions for both adjunctive antipsychotics and adjunctive mood stabilizers was understandably higher for recurrent MDD patients.

Discussion: The present cross-sectional study demonstrated that there is a general tendency toward the prescription of multiple psychotropic drugs for the treatment of MDD inpatients, and that this tendency was more prominent in patients with recurrent episodes. Therefore, a well-designed treatment protocol that is supported by evidence from clinical trials is indispensable for the treatment of MDD in clinical practice.
\end{abstract}

Keywords: major depressive disorders, antidepressant, antipsychotic, mood stabilizer, augmentation, prophylaxis

Received December 26, 2013 / Accepted February 28, 2014 / Published March 28, 2014

Corresponding Author: Akeo Kurumaji, M.D., Ph.D., Section of Psychiatry and Behavioral Sciences, Tokyo Medical and Dental University Graduate School, 1-5-45 Yushima, Bunkyo-ku, Tokyo 113-8519, Japan. Tel: 03-5803-5242, Fax: 03-5803-0135; E-mail: 0724.psyc@tmd.ac.jp 


\section{INTRODUCTION}

Since standard antidepressant therapy enables only a limited number of patients with major depressive disorders (MDD) to achieve a sufficient clinical response and remission [1,2], a stepwise strategy including a change in the antidepressant and the use of adjunctive psychotropic drugs for augmentation [3] is also required in daily clinical practice. While four SSRIs and two SNRIs can be used in Japan, it appears that the possibility that tricyclic antidepressants could be useful for treating MDD that is resistant to the new generation of antidepressants cannot be ruled out. Mood stabilizers, such as lithium carbonate, have an antidepressant augmentation effect [4] as well as a potential prophylactic effect on relapse or recurrence of the MDD episode [5,6]. Atypical antipsychotic drugs have also been proven to be effective augmentation agents [7]. In addition, a small number of studies has shown the superiority of using combinations of antidepressants, such as mirtazapine plus an SSRI or a tricyclic antidepressant plus an SSRI, from the beginning of treatment of MDD [8].

The present study surveyed a range of pharmacological treatments in inpatients with MDD in order to clarify the current state of therapies and the tendency to provide adjunctive pharmacological treatment for MDD during hospital care. The frequency of use of psychotropic drugs in patients with single episodes of MDD was compared to that in patients with recurrent episodes. There was a stronger tendency towards the co-administration of mood stabilizers or antipsychotics in patients with recurrent episodes.

\section{MATERIALS AND METHODS}

The present study included 191 cases of patients with major depressive disorders admitted to the Tokyo Medical and Dental University Medical Hospital from April 2010 to March 2013. Fourteen patients were admitted twice and one patient was admitted three times during this interval due to relapse or recurrence of their depressive disorders. The psychiatrists (authors) diagnosed the inpatients using the criteria of the Diagnostic and Statistical Manual of Mental Disorders, Fourth Edition, Text revision (DSM-IVTR) [9], and assessed the patients using the 17-item Hamilton Depression Rating Scale (HAM-D17) [10]. The demographic data and prescription profiles for all medications at discharge from the hospital were obtained from the medical charts of the patients. Written informed consent was obtained from all the patients, indicating their approval of the use of their clinical information in the study.

\section{STATISTICAL ANALYSIS}

Univariate comparisons of the characteristics of the inpatients with a single episode and the inpatients with recurrent episodes were conducted using chi-squared tests or Fisher's exact tests for the categorical variables and t-tests for the continuous variables. All tests were two-tailed with significance levels of 0.05. A statistical analysis was performed using the Statistical Package for the Social Sciences, version 11.0 (SPSS, Chicago).

\section{RESULTS}

The demographic and clinical characteristics of the inpatients with major depressive disorders are shown in Table 1. There were no statistically significant differences between the patients with a single episode and the patients with recurrent episodes either in their basic characteristics, i.e., age and sex, or in their clinical profiles, i.e., duration of admission, severity of illness, and scores of HAM-D17 at admission and at discharge.

Table 2 shows the frequency of use of each type of psychotropic drug classified into five categories: antidepressants, antipsychotics, mood stabilizers, anxiolytics, and hypnotics. The doses of all of the psychotropic drugs were within the standard clinical dose ranges. Although the prescription rate was significantly higher in patients with recurrent episodes than in patients with single episodes for antipsychotics $(\chi 2=5.06$, d.f. $=1, p=0.019)$, mood stabilizers $(\chi 2=$ 10.661 , d.f. $=1, \mathrm{p}=0.001)$, and hypnotic drugs $(\chi 2=$ 5.600 , d.f. $=1, p=0.018)$, there was no difference between the two groups in the rate of prescription of antidepressants or anxiolytics. Electroconvulsive therapy (ECT) was performed in combination with pharmacotherapy in seven patients with single episodes and 16 patients with recurrent episodes.

There was no difference between the two groups in the frequency of use of each type of antidepressant $(\chi 2$ $=8.333$, d.f. $=5, \mathrm{p}=0.139$ ) (Figure 1). The most commonly used type of antidepressant was selective serotonin reuptake inhibitors (SSRIs) $(\mathrm{n}=80)$, i.e., sertraline, paroxetine, escitalopram, and fluvoxamine, and the next most common was mirtazapine $(n=63)$, followed by trazodone $(n=59)$. The use of tricycles ( $n$ $=33$ ), such as amoxapine, nortriptyline, and clomipramine, serotonin and noradrenaline reuptake inhibitors (SNRIs) $(\mathrm{n}=28)$, i.e., duloxetine and milnacipram, 
and tetracyclics $(\mathrm{n}=33)$, i.e., maprotiline and mianserin, was relatively uncommon. Patients receiving a single antidepressant accounted for only 87 of 191 cases with MDD, and the prescriptions that were written for MDD patients included several different combinations of antidepressants. A large proportion of these patients were treated with mirtazapine or trazodone, and 38 of the 63 patients who were treated with mirtazapine and 52 of the 59 patients who were treated with trazodone also received other antidepressants.

Table 1. Demographic and illness characteristics of inpatients with major depressive disorder

\section{Characteristics}

Gender, female, n (\%)

Age, mean (SD), years

Duration of admission, mean (SD), days

Severity, $\mathrm{n}(\%)$

Mild

Moderate

Severe without psychotic symptoms

Severe with psychotic symptoms

Others

$\begin{array}{lll}\begin{array}{l}\text { Single episode } \\ (\mathrm{n}=71)\end{array} & \begin{array}{l}\text { Recurrent } \\ (\mathrm{n}=120)\end{array} & \mathrm{p} \text { valu } \\ & & \\ 41(58.6) & 82(68.3) & \mathrm{ns} \\ 54.8(16.9) & 54.0(17.0) & \mathrm{ns} \\ 68.2(43.4) & 69.3(47.0) & \mathrm{ns}\end{array}$

ns

$10(8.3)$

$65(53.7)$

$32(26.4)$

$10(8.3)$

$3(2.5)$

HAM-D17 total score, mean (SD)

At admission

$19.6(6.6)$

$19.5(7.3)$

ns

At discharge

$7.0(5.0)$

$6.3(4.4)$

ns

HAM-D17: the 17-item Hamilton Depression Rating Scale. ns: not significant.

Table 2. Frequency of use of psychotropic drugs in patients with major depressive disorders at discharge

\begin{tabular}{llll}
\hline Psychotropic drug & $\begin{array}{l}\text { Single episode } \\
(\mathrm{n}=71)\end{array}$ & $\begin{array}{l}\text { Recurrent } \\
(\mathrm{n}=120)\end{array}$ & $\mathrm{p}$ value \\
\hline Antidepressant & $94.3 \%(67)$ & $91.6 \%(111)$ & $\mathrm{ns}$ \\
Antipsychotic drug & $36.6 \%(26)$ & $53.3 \%(65)$ & $\mathrm{p}<0.05$ \\
Mood stabilizer & $12.7 \%(9)$ & $34.2 \%(41)$ & $\mathrm{p}=0.01$ \\
Anxiolytic drug & $53.5 \%(38)$ & $64.2 \%(77)$ & $\mathrm{ns}$ \\
Hypnotic drug & $71.8 \%(51)$ & $85.8 \%(103)$ & $\mathrm{p}<0.05$ \\
\hline
\end{tabular}

The numbers of patients are shown in parentheses. ns: not significant.

Table 3. Combination drug therapies in inpatients with major depressive disorder at discharge

\begin{tabular}{llll}
\hline Psychotropic drug & $\begin{array}{l}\text { Single episode } \\
(\mathrm{n}=71)\end{array}$ & $\begin{array}{l}\text { Recurrent } \\
(\mathrm{n}=120)\end{array}$ & $\mathrm{p}$ value \\
\hline Antidepressant & $52.1 \%(37)$ & $34.2 \%(41)$ & $<0.05$ \\
Antidepressant + Antipsychotics & $28.2 \%(20)$ & $28.3 \%(34)$ & $\mathrm{ns}$ \\
Antidepressant + Antipsychotics + Mood stabilizer & $5.6 \%(4)$ & $17.5 \%(21)$ & $<0.05$ \\
Antidepressant + Mood stabilizer & $8.5 \%(6)$ & $10.8 \%(13)$ & $\mathrm{ns}$ \\
Antipsychotics + Mood stabilizer & $0.0 \%(0)$ & $5.0 \%(6)$ & $\mathrm{ns}$ \\
Others & $5.6 \%(4)$ & $4.2 \%(5)$ & $\mathrm{ns}$ \\
\hline
\end{tabular}

The numbers of patients are shown in parentheses. The differences between patients with single episodes and patients with recurrent episodes in the distribution of combination drug therapies were analyzed using the chi-squared test $(\chi 2=12.325$, d.f. $=5, p=0.031)$. ns: not significant. 


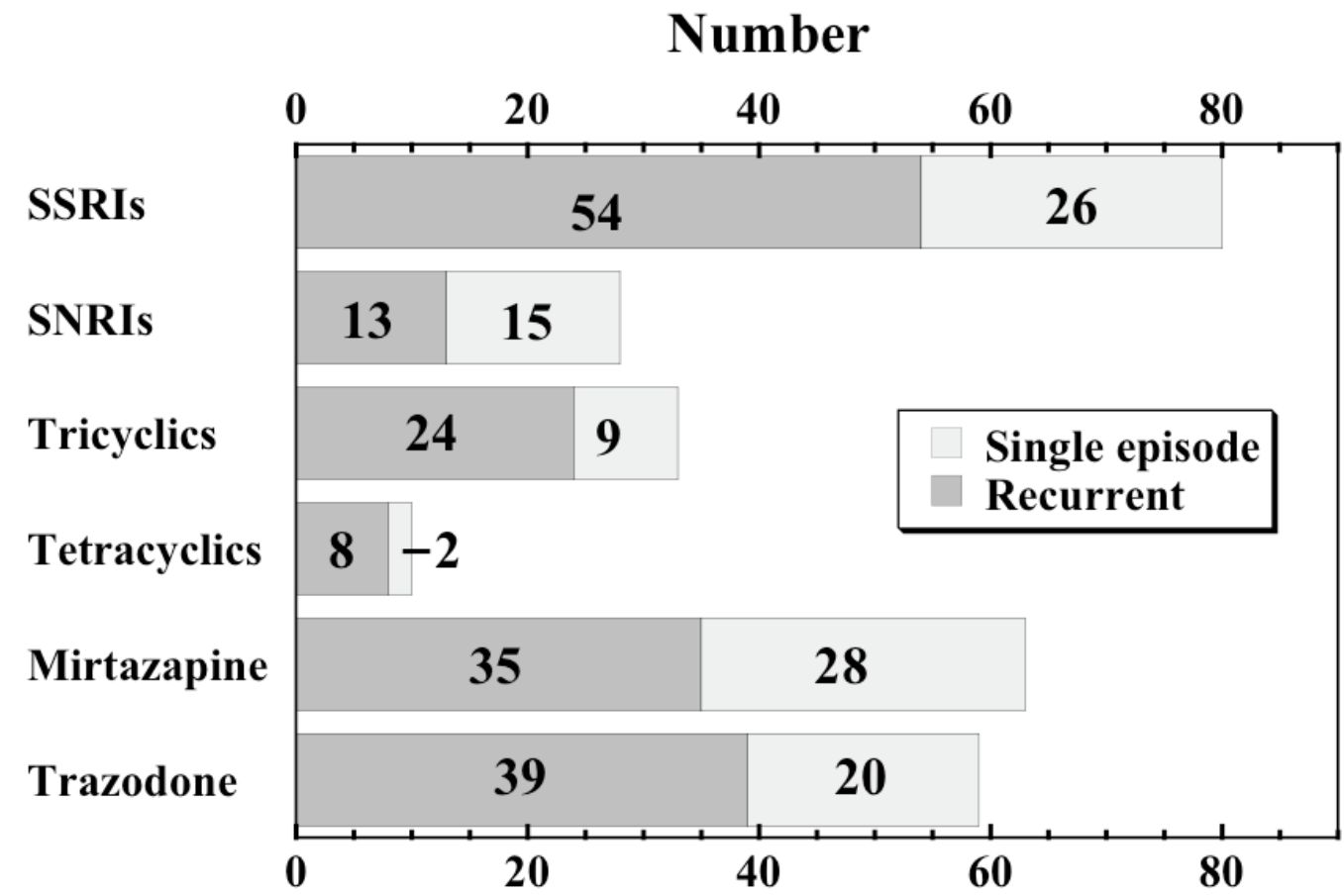

Figure 1. The frequency of use of antidepressants in inpatients with major depressive disorders with single and recurrent episodes.

Data are divided into four groups and two antidepressants. The serotonin reuptake inhibitors (SSRIs) included sertraline, paroxetine, escitalopram, and fluvoxamine, and the serotonin and noradrenaline reuptake inhibitors (SNRIs) were duloxetine and milnacipram. Five tricyclic antidepressants, i.e., amoxapine, amitriptyline, nortriptyline, clomipramine, and imipramine, and two tetracyclics, i.e., maprotiline and mianserin, were used for the major depressive disorders. There was no difference in the frequency of use of each type of antidepressant between the single and recurrent types of $\operatorname{MDD}(\chi 2=8.333$, d.f. $=5$, $\mathrm{p}=$ $0.139)$.
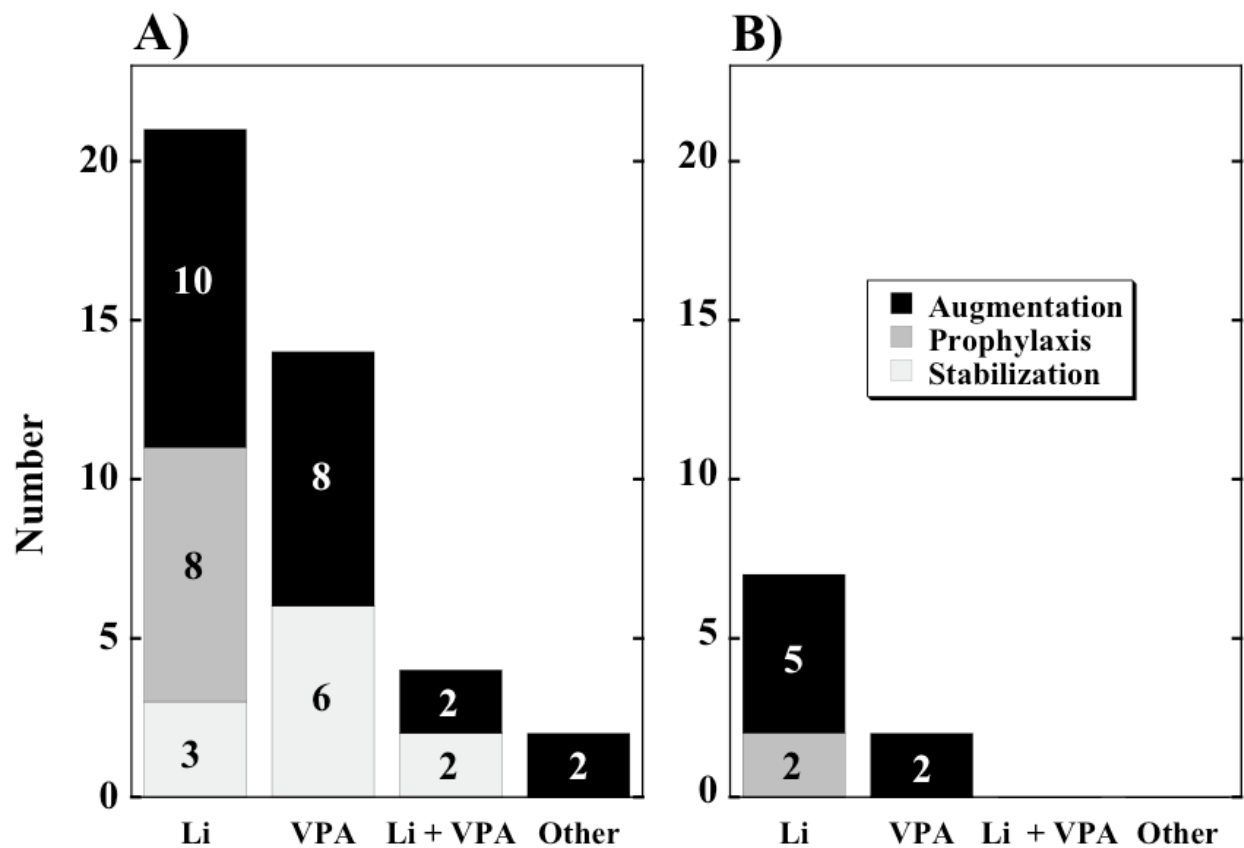

Figure 2. The adjunctive use of mood stabilizers in inpatients with major depressive disorder.

Forty-one inpatients with recurrent episodes (A) and nine inpatients with a single episode (B) were prescribed mood stabilizers; the pattern of prescription was classified into four groups, namely lithium (Li) alone, valproic acid (VPA) alone, a combination of Li and VPA, and other (Li plus carbamazepine or lamotrigine).

Mood stabilizers were administered to the MDD patients for therapeutic purposes, i.e., augmentation of antidepressants, prevention from recurrence of depressive episodes and stabilization of manic symptoms in a subthreshold level of the hypomanic episode [9]. The frequency of use of mood stabilizers in the MDD patients, stratified by the type of drug and the objective of use, is shown in Figure 2. With regard to augmentation, lithium carbonate targeted the core symptoms of depression, such as depressed mood and 
loss of energy, while valproic acid was mainly given for patients who easily switch from normothymia to a depressive state. Lithium carbonate was also used for prophylaxis in eight patients with recurrent episodes and two patients with single episodes after ECT. Mood stabilizers were used to treat manic symptoms in eight patients with recurrent episodes.

A profile of the psychotropic combinations administered to all the MDD patients is shown in Table 3. A statistically significant difference was observed in the frequency of each type of combination between patients with single episodes and patients with recurrent episodes $(\chi 2=12.325$, d.f. $=5, p=0.031)$. The use of a combination of three types of psychotropic drugs, i.e., antidepressants, mood stabilizers, and antipsychotic drugs, was significantly higher in patients with recurrent episodes, and the prescription of an antidepressant with no adjunctions was substantially lower.

\section{DISCUSSION}

The present study demonstrated that inpatients with recurrent episodes more frequently received antipsychotic drugs, mood stabilizers, and hypnotics at discharge from the hospital than patients with single episodes, even though there were no differences between the two types of patients in the severity of illness, outcome of treatment, or duration of hospital stay. Naturally, the rate of use of both antipsychotics and mood stabilizers as add-on therapy to antidepressants was higher in patients with recurrent episodes than in patients with single episodes.

The effectiveness of the augmentation of antidepressants with lithium has been demonstrated by a meta-analysis [4]. It appears that valproic acid was also effective for augmentation targeting the vulnerable mood state in the present study. In addition to the augmentation of antidepressants, mood stabilizers were administered to inpatients with MDD with recurrent episodes not only for the prevention of recurrence of the MDD episode, but also for stabilizing a mixed depressed state. It has been reported that add-on treatment with lithium as an antidepressant after ECT had a prophylactic effect on the relapse or recurrence of the MDD episode $[5,6]$. We also reported the efficacy in two cases of elderly patients with recurrent MDD [11]. Attention to the subthreshold hypomanic state from the perspectives of diagnosis and treatment is required in clinical practice [12-14]. In the present study, several inpatients with recurrent episodes who exhibited manic symptoms, such as irritability, talkativeness, or increased activity, adjunctively received mood stabilizers.

This study also shows that more than half of all patients received a combination of antidepressants during the treatment of the episode of MDD. The majority of patients who received a combination of antidepressants received mirtazapine or trazodone. The superiority of combination therapy with mirtazapine and an SSRIs to an SSRI alone for achieving remission has been proven in a few studies $[15,16]$. A low-dose $(25-75 \mathrm{mg} /$ day) of trazodone was used in the present study due to its anxiolytic action and its ability to normalize sleep patterns in depressed patients [17]. Taking into account the high prescription rate of hypnotic drugs in patients with recurrent MDD, the resistance of insomnia to standard antidepressant therapy may be one of the practical problems encountered in the therapeutic approach to remission in MDD inpatients.

Antipsychotic drugs were prescribed to inpatients with MDD for the augmentation of antidepressants, which was more common in patients with recurrent episodes than in patients with single episodes in the present study. The effectiveness of atypical antipsychotics, i.e., quetiapine, olanzapine, risperidone, and aripiprazole, as add-on therapy in MDD has been supported by a meta-analysis of placebo-controlled randomized trials [7]. The availability of antipsychotics as an on-label use in Japan [18] would allow evidence to be gathered for determining the efficacy of augmentation.

In conclusion, although this present cross-sectional study has a number of limitations, such as an institutional bias, this study shows that there is a general tendency towards the use a combination of psychotropic drugs for the treatment of MDD, and that this tendency was more pronounced in patients with recurrent episodes than in patients with single episodes, and was associated with a resistance to pharmacotherapy and other factors such as prophylaxis and the complexity of the symptoms. An adequate combination of psychotropic drugs seems to be indispensable for MDD inpatients with moderate or severe intensity to achieve remission and remain in a state of remission.

\section{ACKNOWLEDGEMENTS}

This study was partly supported by research grants from the Ministry of Health, Labor and Welfare (Japan), and a Grant-in-Aid for Science Research from the Ministry of Education, Science, Culture and Sports (Japan). 


\section{CONFLICTS OF INTEREST}

All authors declare that they have no conflicts of interest.

\section{REFERENCES}

[1] Casacalenda N, Perry JC, Looper K. Remission in major depressive disorders: a comparison of pharmacotherapy, psychotherapy and control conditions. Am J Psychiatry 2002; 159: 1354-1360.

[2] Fava M. Diagnosis and definition of treatment-resistant depression. Biol Psychiatry 2003; 53: 649-659.

[3] Rush AJ, Trivedi MH, Wisniewski SR, Nierenberg AA, Stewart JW, Warden D, Niederehe G, Thase ME, Lavori PW, Lebowitz BD, McGrath PJ, Rosenbaum JF. Acute and longer-term outcomes in depressed outpatients requiring one or several treatment steps: a STAR*D report. Am J Psychiatry 2006; 163: 1905-1917.

[4] Bauer M, Adli M, Bschor T, Pilhatsch M, Pfennig A, Sasse J, Schmid R, Lewitzka U. Lithium's emerging role in the treatment of refractory major depressive episodes: augmentation of antidepressants. Neuropsychobiology 2010; 62: 36-42.

[5] Sackeim HA, Haskett RF, Mulsant BH, Thase ME, Mann JJ, Pettinati HM, Greenberg RM, Crowe RR, Cooper TB, Prudic J. Continuation pharmacotherapy in the prevention of relapse following electroconvulsive therapy: a randomized control trial. JAMA 2001; 285: 1299-1307.

[6] Kellner CH, Knapp RG, Petrides G, Rummans TA, Husain MM, Rasmussen K, Mueller M, Bernstein HJ, O'Connor K, Smith G, Biggs M, Bailine SH, Malur C, Yim E, McClintock S, Sampson S, Fink M. Continuation electroconvulsive therapy vs. pharmacotherapy for relapse prevention in major depression. Arch Gen Psychiatry 2006; 63: 1337-1344.

[7] Nelson JC, Papakostas, GI. Atypical antipsychotic augmentation in major depressive disorder: a meta-analysis of placebo-controlled randomized trials. Am J Psychiatry 2009; 166: 980-991.

[8] Carpenter LL, Yasmin S, Price LH. A double-blinded, placebo-controlled study of antidepressant augmentation with mirtazapine. Biol Psychiatry 2002; 51: 183-188.
[9] American Psychiatry Association. Diagnostic and Statistical Manual of Mental Disorders DSM-IV-TR Fourth Edition. $4^{\text {th }}$ ed. Washington, DC: American Psychiatric Publishing Inc; 2000.

[10] Hamilton M. A rating scale for depression. J Neurol Neurosurg Psychiat 1960; 23: 56-62.

[11] Ishikawa H, Atsuta H, Ohshima K, Nishikawa T, Kurumaji A. An effectiveness of adjunctive treatment with lithium upon remission of elderly-onset psychotic depressive disorders (two cases) after ECT. Psychiatry 2009; 15: 381-386. (In Japanese).

[12] Benazzi G. Bipolar disorder-focus on bipolar II disorder and mixed depression. Lancet 2007; 369: 935-945.

[13] Goldberg JF, Perlis RH, Bowden CL, Thase ME, Miklowitz DJ, Marangell LB, Calabrese JR, Nierenberg AA, Sachs GS. Manic symptoms during depressive episodes in 1,380 patients with bipolar disorder: findings from the STEP-BD. Am J Psychiatry 2009; 166: 173-181.

[14] Frank NR. Subthreshold bipolarity: diagnostic issues and challenges. Bipolar Disord 2011; 13 : 587-603.

[15] Rocha FL, Fuzikawa C, Riera R, Ramos MG, Hara C. Antidepressant combination for major depression in incomplete responders-a systematic review. J Affect Disord 2013; 144: 1-6.

[16] Rocha FL, Fuzikawa C, Riera R, Hara C. Combination of antidepressants in treatment of major depressive disorder. J Clin Psychopharmacol 2012; 32: 278-281.

[17] Fagiolini A, Comandini A, Dell'Osso MC, Kasper S. Rediscovering trazodone for the treatment of major depressive disorder. CNS Drugs 2012; 26: 1033-1049.

[18] Kamijima K, Higuchi T, Ishigooka J, Ohmori T, Ozaki N, Kanba S, Kinoshita T, Koyama T, ADMIRE Study Group. Aripiprazole augmentation to anti depressant therapy in Japanese patients with major depressive disorder: A randomized, double-blind, placebocontrolled study (ADMIRE study). J Affect Disord 2013; 151: 899-905. 